\title{
Enhancing nonclassical bosonic correlations in a quantum walk network through experimental control of disorder
}

\author{
Alessandro Laneve,,${ }^{1, *}$ Farzam Nosrati $\odot,{ }^{2,3, \dagger}$ Andrea Geraldi, ${ }^{1}$ Kobra Mahdavipour $\odot,{ }^{2,3}$ Federico Pegoraro, \\ Mahshid Khazaei Shadfar $\odot,{ }^{2,3}$ Rosario Lo Franco $\odot,{ }^{4}$ and Paolo Mataloni ${ }^{1}{ }^{1}$ \\ ${ }^{1}$ Dipartimento di Fisica, Sapienza Università di Roma, Piazzale Aldo Moro, 5, I-00185 Roma, Italy \\ ${ }^{2}$ Dipartimento di Ingegneria, Università di Palermo, Viale delle Scienze, Edificio 9, 90128 Palermo, Italy \\ ${ }^{3}$ INRS-EMT, 1650 Boulevard Lionel-Boulet, Varennes, Québec J3X 1S2, Canada \\ ${ }^{4}$ Dipartimento di Ingegneria, Università di Palermo, Viale delle Scienze, Edificio 6, 90128 Palermo, Italy
}

(Received 9 February 2021; accepted 14 July 2021; published 10 September 2021)

\begin{abstract}
The presence of disorder and inhomogeneities in quantum networks has often been unexpectedly beneficial for both quantum and classical resources. Here we experimentally realize a controllable inhomogenous quantum walk (QW) dynamics, which can be exploited to investigate the effect of coherent disorder on the quantum correlations between two indistinguishable photons. Through the imposition of suitable disorder configurations, we observe two-photon states that exhibit an enhancement in the quantum correlations between two selected modes of the network, compared to the case of an ordered QW. Different configurations of disorder can steer the system toward different realizations of such an enhancement, thus allowing spatial and temporal manipulation of quantum correlations between remote modes of QW networks.
\end{abstract}

DOI: 10.1103/PhysRevResearch.3.033235

\section{INTRODUCTION}

A thorough characterization of genuine quantum traits is crucial to understand the boundary between classical and quantum phenomena [1] and to perform quantum information tasks [2]. To this aim, several quantification methods have been introduced to faithfully identify the presence of quantum (nonclassical) resources such as entanglement [3], coherence [4], discord [5], joint measurability [6], steering [7], or thermal operations [8] in the case of composite systems.

Indistinguishability of quantum identical particles $[9,10]$ has also been revealed as a useful nonclassical resource. From an operational point of view, particles are so-called indistinguishable if they are in the same mode with respect to a characterization via two-particle interference [11]. From a broader perspective, the indistinguishability concept is related to a given set of quantum measurements [12]. In fact, indistinguishability plays a fundamental role in raising quantum processes, such as many-body interference [11,13], entanglement generation [9,10,14-17], quantum teleportation [17], quantum metrology [18,19], quantum coherence [20-22], quantumness protection [12,23,24], quantum key distribution $[25,26]$, and the high state complexity exploited by boson sampling algorithms [27,28].

\footnotetext{
*alessandro.laneve@uniroma1.it

†farzam.nosrati@unipa.it

Published by the American Physical Society under the terms of the Creative Commons Attribution 4.0 International license. Further distribution of this work must maintain attribution to the author(s) and the published article's title, journal citation, and DOI.
}

In this context, it is important to understand how quantum features based on indistinguishability behave in a dynamical framework, specifically in the case of bosons propagating through a nonhomogeneous system. For a large variety of systems, the disorder plays a detrimental role because it drives the system into decoherence [29]. Contrarily, for some systems, the disorder can enhance physical properties such as coherent transport [30], quantum algorithms speedup [31], and quantum correlations [32-35]. These effects commonly appear due to the interaction with an external environment, though not always featuring a back-action mechanism [36,37].

A suitable theoretical platform to perform such a study is represented by quantum walk (QW), which provides a very general coherent propagation model: At variance with classical random walks, QWs are able to preserve genuine nonclassical features such as superposition, interference, and entanglement [38-40]. QWs provide powerful models to describe energy transport phenomena in different types of systems like photosynthetic complexes [30,41], or solid state ones, as in the case of Luttinger liquids [42].

It has been shown that adjustable disorder plays a significant role in the evolution of quantum walker in which the ballistic growth can become anomalous, classical, or localized [43-47]. The dynamics of a quantum walker is intimately connected to its nonclassical features. The way quantum-correlated walkers, realized by photon pairs, evolve in a homogeneous optical lattice has been investigated, highlighting the different behavior of distinguishable or indistinguishable photons [48-52]. Moreover, the spreading pattern of the quantum walker(s) can be modified through various types of disorder [44,46,47,53].

At variance with previous studies so far [35], it still remains to find strategies for enriching two-particle quantum 
correlations via disorder control. Here we fill this gap through the experimental observation of the propagation of two indistinguishable photons (biphoton) in a one-dimensional inhomogeneous discrete time QW (1D DTQW). In the absence of disorder, the initial quantum correlation in the system features a global and local decrease as the evolution proceeds. Contrarily, we achieve to find certain disorder configurations that partially preserve the initial quantum correlation of a biphoton propagating in a discrete QW network, after a given number of evolution steps. The presented photonic setup is therefore an experimental platform capable of enhancing the biphoton quantum correlation between two selected modes of the QW network via disorder realizations, without any interaction with auxiliary systems. Our findings unambiguously prove that the presence of disorder fosters the dynamical enhancement of biphoton quantum correlations in a controllable fashion, paving the way to its potential employment in quantum information scenarios.

\section{THEORETICAL FRAMEWORK}

The 1D QW model consists of one or more walkers coherently moving along the discretized sites of a line [39]. In general, the state of the system can always be written as a superposition of the QW modes $|\Psi(t)\rangle=\sum_{k} \alpha_{k}(t)|k\rangle$, where each mode $|k\rangle:=|x\rangle|\sigma\rangle$ is defined by both position $|x\rangle$ and its coin $|\sigma\rangle=\{|L\rangle,|R\rangle\}$, and amplitudes $\alpha_{k}(t)$ depend on the past evolution of the walker. Therefore, the singlestep evolution can be written as $|\Psi(t+1)\rangle=\sum_{k} \hat{U} \alpha_{k}(t)|k\rangle$, where $\hat{U}=\hat{S} \cdot(\hat{I} \otimes \hat{C})$ is the one-step evolution operator with $\hat{S}=\sum_{x}|x+1\rangle\langle x|\otimes| L\rangle\langle L|+| x-1\rangle\langle x|\otimes| R\rangle\langle R|$ being the shift operator that moves the walker according to the coin state, $\hat{I}$ is the position identity operator, and $\hat{\mathcal{C}}$ is the coin operator, which in our case reads $\hat{\mathcal{C}}=\frac{1}{\sqrt{2}}(|L\rangle\langle L|+i| L\rangle\langle R|+$ $i|R\rangle\langle L|+| R\rangle\langle R|)$. We also allow for the presence of stepposition-dependent phases $\phi_{k}(t)$, which are responsible for the dynamical disorder that the quantum walker experiences, according to the so-called $p$-diluted model [46].

In this disorder framework, the single-step evolution of the quantum state of the system can be written as

$$
|\Psi(t+1)\rangle=\sum_{k} e^{i \phi_{k}(t)} \hat{U} \alpha_{k}(t)|k\rangle,
$$

where step-position-dependent phases $\phi_{k}(t)$ are chosen out of two choices 0 or $\pi$. Here we assume that the phases experienced by the quantum walker during its evolution are independently and randomly generated. In order to generate a random phase map, a Bernoulli process determines whether the phase shift at step $t$ and position $x$ is $\phi=0$ [with a certain probability $\left.\left(1-\frac{p}{2}\right)\right]$ or $\phi=\pi$ (with probability $\frac{p}{2}$ ). In the end, a phase map is a set of matrices $\left\{\Phi_{p}(t)\right\}_{t=0, \ldots t_{\max }}$ that describe all the phases imposed on the walker during the QW process with a given fixed $p$. This results in a network structure underlying the QW evolution, clearly represented by Fig. 1. On average, photons will experience a $\frac{p}{2}$ percentage of "flipped" phases during the quantum evolution. It is worth noting that the evolution stays coherent; in fact, there is no decoherence in this framework: If the initial state is pure, the final state stays so. In the following, we especially focus on the single phase maps that enhance the quantum correlation

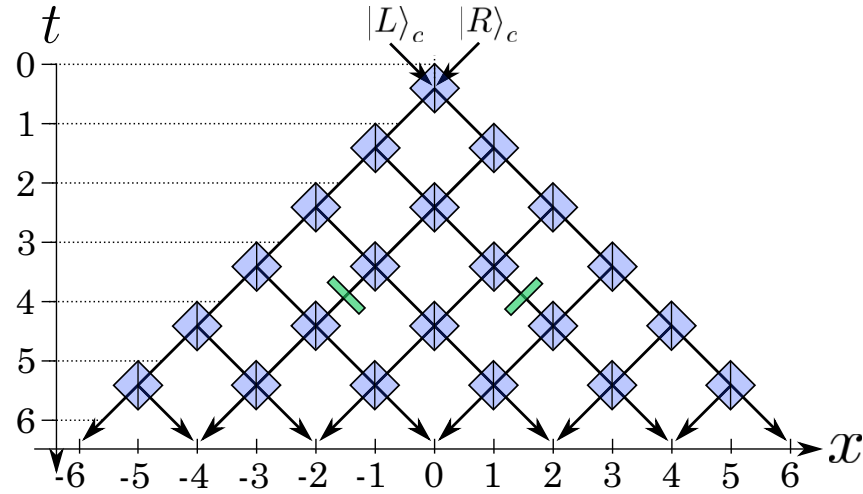

FIG. 1. Network representation of a disordered $Q W$ according to p-diluted model. The green bars represent $\pi$ phases on the path which can be added to or removed from the network. Coin and shift operations are represented here by BSs (shaded cyan squares). The $|R\rangle_{c}$ and $|L\rangle_{c}$ states are indicated.

between the walkers; in that case, the average level of disorder $p$ is no more a relevant quantity.

In order to study the effect of disorder over nonclassical bosonic correlations in a QW dynamics, we consider two indistinguishable photon walkers as input. This choice is strategical since, unlike states of distinguishable photons, the state of an indistinguishable photon pair (biphoton) $\left|\Psi^{(2)}\right\rangle=\left|k_{1}, k_{2}\right\rangle\left(\left|k_{i}\right\rangle=\left|x_{i}\right\rangle\left|\sigma_{i}\right\rangle\right)$ exhibits intrinsic quantum correlations $[54,55]$. The two-particle evolution is obtained by applying $\hat{U} \otimes \hat{U}$ to the initial state. Inspired by classical intensity correlation of light, one way to measure the nonclassicality of the correlation between two detected outputs is by violating the inequality $[48,49]$,

$$
V_{i j}=\frac{2}{3} \sqrt{\Gamma_{i i} \Gamma_{j j}}-\Gamma_{i j}<0,
$$

where $\Gamma_{i j}$ is the probability of finding a photon in mode $i$ and the other one in $j$, namely, the probability of measuring a coincidence between modes $i$ and $j$. Inequality (2), in fact, stands for classically correlated light and its violation is assumed to witness and quantify the presence of quantum correlations, as a signature of photon bunching $[48,49,52]$. In a discrete network, the violation of the inequality between two modes can be considered as evidence of quantum correlation which contains part of the total correlation information (see Appendix A for a detailed discussion of $V_{i j}$ interpretation).

\section{NUMERICAL RESULTS}

Preliminary simulations were carried out in an ideal $p$ diluted framework: Two indistinguishable photons traveling a bulk-optics 1D DTQW provided with space-time disorder.

We studied the average behavior of the violation matrix by averaging over many different evolution realizations with randomness $p$. The average probability distribution is used to obtain the output violation matrices. We show the violation matrix of an indistinguishable photon pair after 15 steps for the ordered case Fig. 2(a), corresponding to the disorder level $p=0$ and the completely disordered one Fig. 2(b) featured by maximal randomness $p=1$. In this case, the disorder level $p$ 


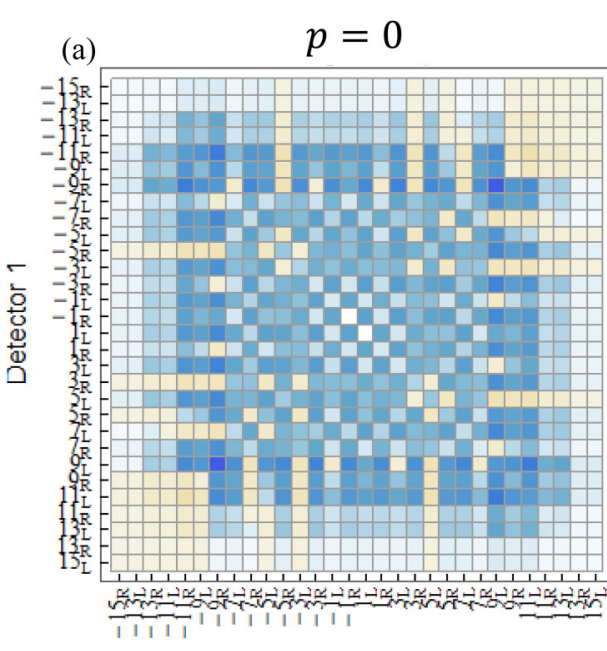

Detector 2

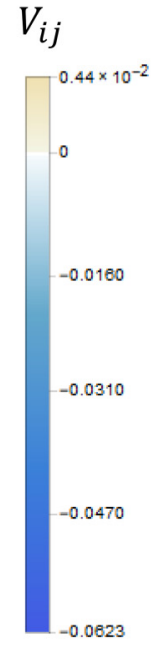

(2)

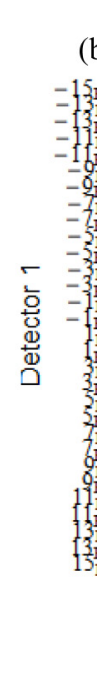

(b)

$p=1$
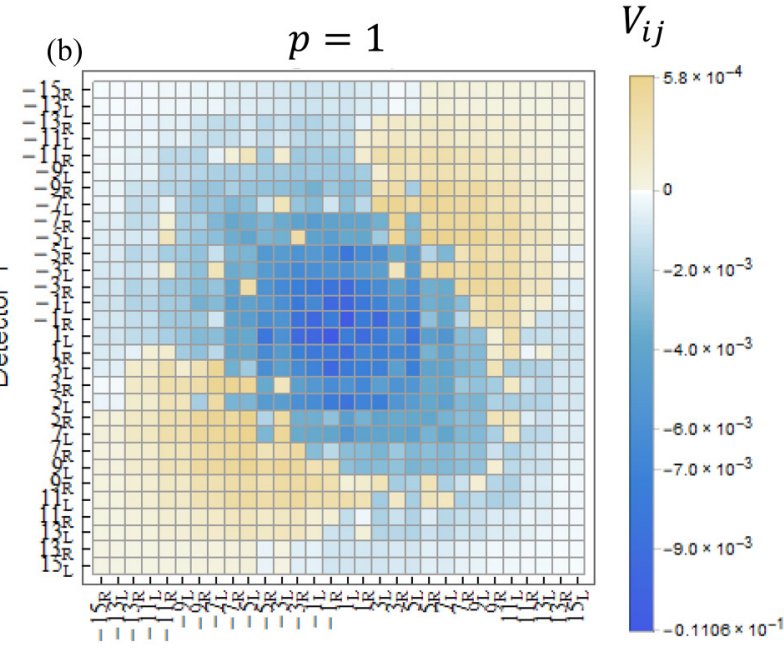

Detector 2

FIG. 2. Numerical simulation of violation matrices. Step 15 matrices of the $V_{i j}$ values of indistinguishable photons in the (a) ordered case $(p=0)$ and the (b) completely disordered one $(p=1)$. The disordered matrix has been computed by averaging over $10^{4}$ disorder configurations.

is a relevant quantity since it indicates the average quantity of disorder imposed on the evolution. As can be seen, violations are present both in the ordered and in the disordered case, though there is an evident migration of the violating values toward the matrix tails. We report in Fig. 3 the values of probability $\Gamma_{i j}$ of finding a coincidence between two photons emerging from modes $i$ and $j$. Indeed, in the completely disordered case, a strong funneling of population toward central modes is present, while quantum correlations disappear in the central region. This phenomenon may nourish the idea that correlations specifically generated by the QW dynamics are classical and may even smother the underlying nonclassical correlations [56,57], especially in the completely disordered case, when the QW dynamics emulates the one of a classical random walk.

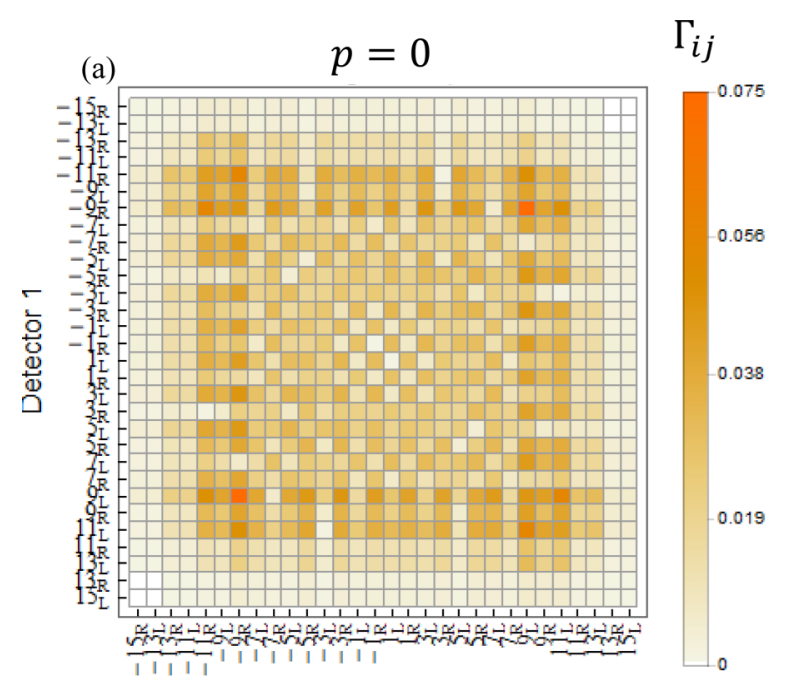

Detector 2
Here, the dynamical average behavior of quantum correlation has been studied through total violation, defined as the sum of all the $V_{i, j}$ of the violating coincidences. It has been considered as a measure of the total quantum correlation present in the system. The normalized trend in function of the disorder level is reported in Fig. 4 for different evolution time lengths. total violation has a decreasing trend as $p$ increases, which can be seen as a consequence of the migration picture described above. Since violations are bound to appear only between scarcely populated modes, the global quantum correlations diminishes.

In general, the average evolution appears naturally featured by a decrease of global nonclassicality in time. Nevertheless, this nonclassicality degradation can be challenged by specific single realizations of disorder. In order to find such phase

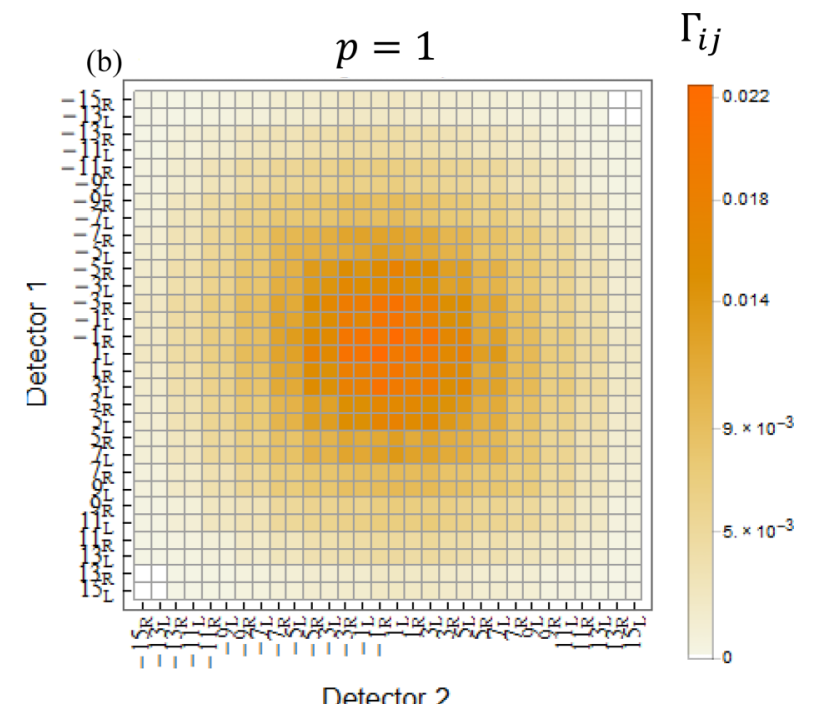

Detector 2

FIG. 3. Numerical simulation of coincidences matrices. Step 15 output distributions of indistinguishable photons in the (a) ordered case $(p=0)$ and the completely (b) disordered one $(p=1)$. The disordered matrix has been computed by averaging over $10^{4}$ disorder configurations. 


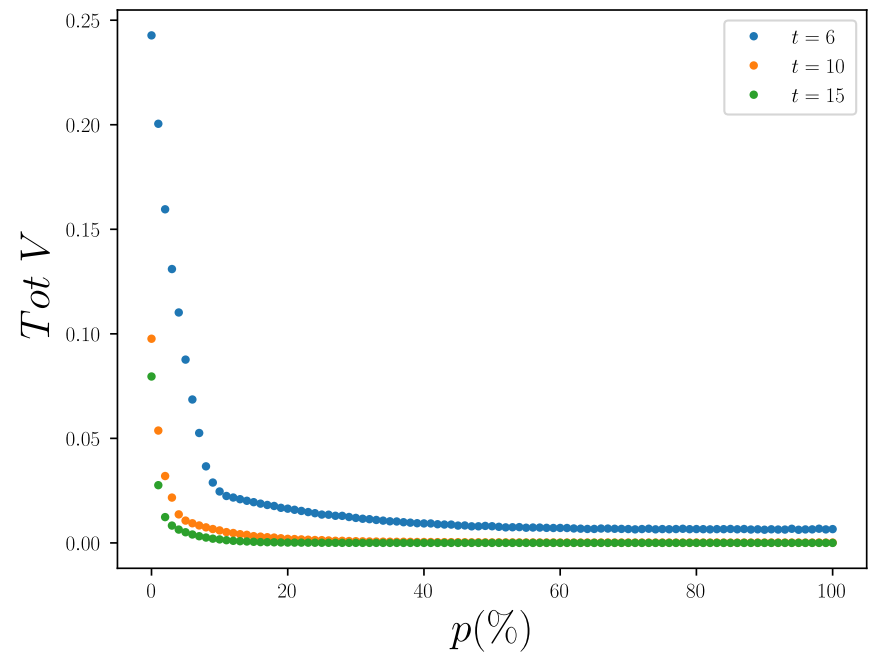

FIG. 4. Numerical simulation of total violation trends. Step 15, step 10, and step 6 plot of the average total violation, computed over $10^{4}$ disorder configurations, as a function of the disorder level $p$.

maps, we adopt a random search protocol: We simulate the evolution with $10^{4}$ different phase maps for each step number $t$ up to 30 steps for a total amount $3 \cdot 10^{5}$ of explored configurations. These configurations are generated for a fixed level for disorder, but, since we focus on specific realizations, the average level of disorder becomes irrelevant. We compute the $V_{i j}$ between each pair of output modes for each simulated probability distribution, obtaining the corresponding violation matrices. Then, the entire set of $V_{i j}$ elements corresponding to any combination of $i$ and $j$ modes is reported. Now we can compare the values for any pair $(i, j)$ and for each simulated phase map at a given step to find the maximum achievable violation (MAV), i.e., the maximum positive value of $V_{i j}$ which could be achieved at that given step. In this way, it is also possible to recognize the specific phase maps generating the MAVs, which can then be experimentally exploited. A more detailed numerical study about the conditions for emerging of MAVs is reported in Appendix B.

(a)

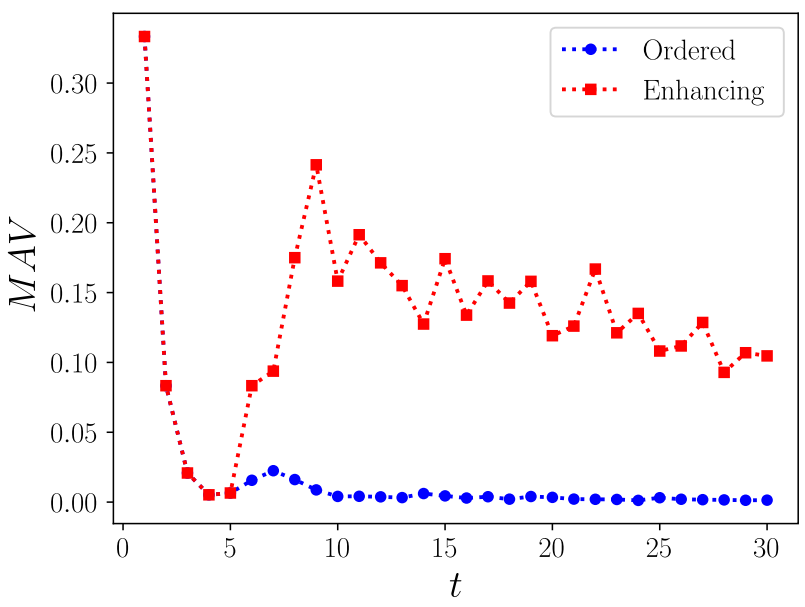

Summarizing, for each configuration, the procedure consists of comparing maximum positive violations of inequality (2) at step $t$. This also allows to know where and when it is beneficial to apply the $\pi$ phase shift within the QW network to identify and select enhancing phase maps. Simulation results are shown in Figs. 5(a) and 5(b), respectively. We report the MAV as a function of the number of steps, and the stepwise trend of the maximum achievable total violation, defined as the sum of all the positive values $V_{i j}$ of the considered violation matrix (named here as total quantum correlation). Since the explored configurations may not cover the entire set of possible disorder patterns, the results cannot be considered absolutely optimal, but rather enhancing in comparison with the ordered case. Nevertheless, the analysis highlights that disorder helps to retrieve quantum correlations after a specific step of QW, both for MAV and for total violation, suggesting that the two quantities are related although not bound to be maximized together. From numerical results, we can conclude that disorder, acting through mere interference, significantly modifies the evolution of the walker, not only reshaping the probability distribution but also affecting the amount of quantum correlation between the photons. As a consequence, disorder may enable enhancement of the quantum correlation of a bipartite system. It is worth noting that no violations are observed, whatever the phase map, when a state of distinguishable photons is employed [49].

\section{EXPERIMENTAL SETUP}

The experimental setup consists of a bulk-optics multipass double Sagnac interferometer (SI) in which inhomogeneities, described by the phases $\phi_{k}(t)$, can be addressed independently both in step number and evolution mode. The bulk-optics setup shown in Fig. 6 is analogous to a chain of intrinsically phase-stable Mach-Zehnder interferometers (MZIs), each provided with an individually tunable phase shifting. The additional exploitation of the $z$ direction allows to effectively realize a beam splitter (BS) network, such as the one in Fig. 1, which reproduces a 1D DTQW dynamics where the coin and

FIG. 5. Numerical simulation compression between ordered and enhancing disordered $Q W$. (a) MAV and (b) maximum achievable total violation versus the number of steps (discrete time) $t$ for the ordered (blue circles) and enhancing disordered (red squares) QW. 


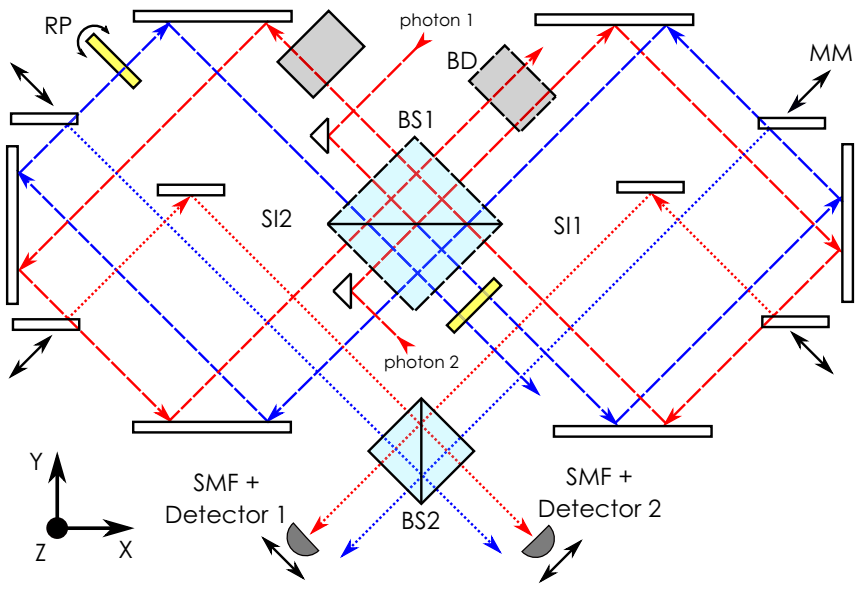

FIG. 6. The $2 D$ sketch of the experimental setup. BS, beam splitter; $\mathrm{BD}$, beam displacer; RP, rotating glass plates; MM, moving mirror; S1, Sagnac interferometer 1; S2, Sagnac interferometer 2. Blue and red beams circulate in opposite directions and impinge on the $B S 1$ in the same horizontal point but at different heights along the $\mathrm{z}$ direction, due to the effect of BDs.

position states are both encoded in the input and output direction of photons with respect to the BS. Thanks to the particular geometry of the implementation, each propagation mode of the QW at each given step has a specific position in the plane transverse to the propagation direction. Therefore, the phase shifts can be independently addressed in each mesh of the QW by the simple insertion of rotating glass plates (RP) along the propagation path. The output state can be measured through a set of moving mirrors (MMs), intercepting and extracting from the setup-only modes of the selected step $t_{j}$. Also, the previous propagation steps $t<t_{j}$ are not affected in any way by the measurement procedure. The extracted radiation is then coupled to a single-mode fiber and measured (for further details on the setup, see Refs. [46,58]). Using couplers to collect the extracted photons, we can measure coincidences between all possible modes at each step and experimentally reconstruct the two-photon probability distribution. The single-photon source is described in detail in Appendix B, together with further specifics of the experimental setup.

\section{EXPERIMENTAL RESULTS}

To experimentally verify disorder-induced changes in the violation matrix, we measure both ordered and disordered evolution QW output distributions. In fact, based on the simulation study displayed in Fig. 5, the first quantum correlation enhancement, due to disorder, shall occur at the sixth step of QW. Therefore, the output violation matrices for optimal phase maps are measured up to the sixth step. Since there is no enhancement until the fifth step, the corresponding optimal phase maps can be considered equivalent to the ordered one, while for the sixth step it is possible to find many specific disorder configurations enhancing the nonclassicality in the correlation between two chosen output modes for both the ideal case and the accounting for experimental parameters. The phase map selected for the experimental implementation features phase shifters at step $t=4$, position $x=-2$ with coin $\sigma=L$, and $x=2$ with coin $\sigma=R$ set to $\pi$, while all the others are left to zero; the corresponding experimental output violation matrix is shown in Fig. 7, compared with the expected one, where the mode $|k\rangle=|x\rangle|\sigma\rangle$ is indicated by $x_{\sigma}$. A strong quantum correlation peak appears at modes $\left(2_{L},-2_{R}\right)$ and $\left(-2_{R}, 2_{L}\right)$ confirming the expectation. As a further relevant result, the experimental stepwise trend for MAV is shown in Fig. 8(a), in comparison with the expected enhanced one obtained by numerical analysis, taking into account experimental constraints. They are plotted together with the ordered case trend to provide a clear display of the beneficial effect of the nonhomogeneous evolution. Theoretical patterns are shown up to the 10th step as a reference. The corresponding trends for the total violation computed over the same output distributions are also reported in Fig. 8(b). Simulations of the MAV values in the ordered case show that the quantum
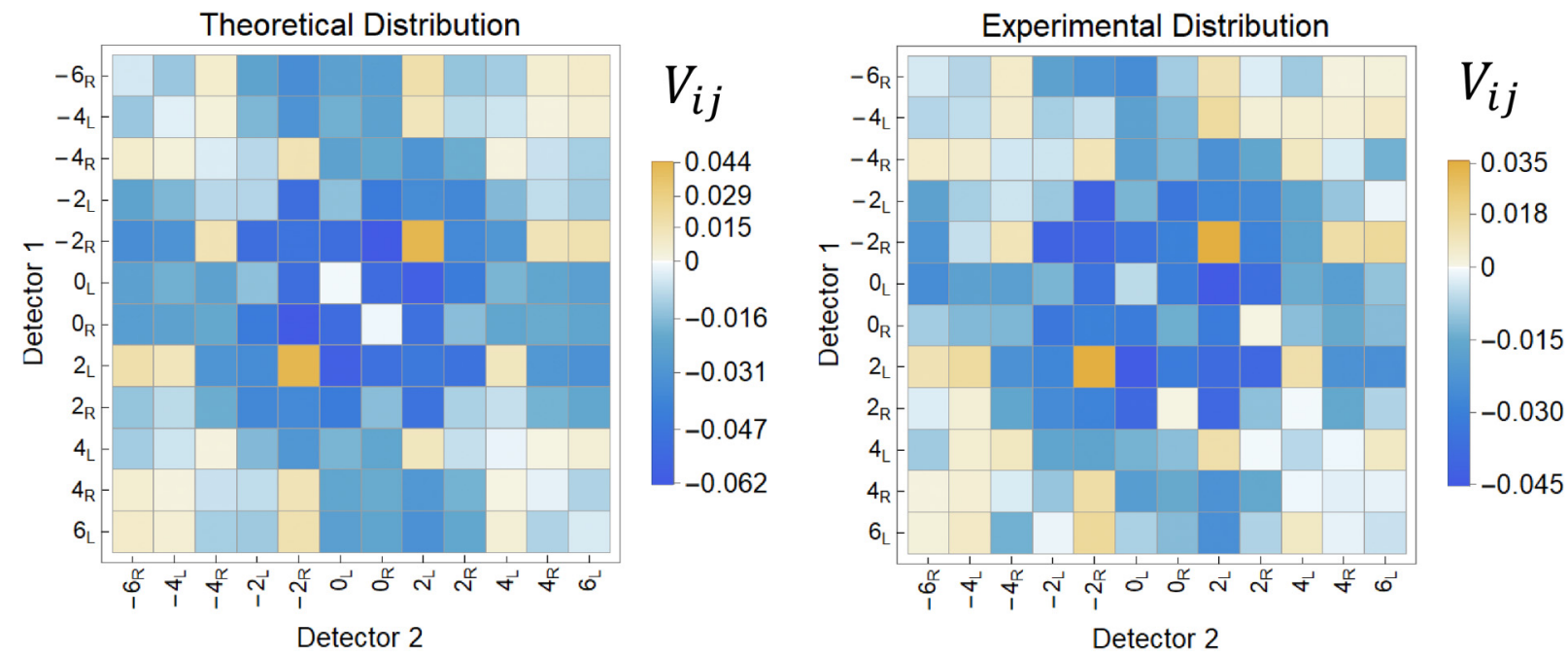

FIG. 7. Comparison between the theoretical and the experimental violation matrices at the sixth step for an enhancing disorder configuration. Numerical simulations are performed taking into account experimental parameters. The expected peak in the value of $V$ is experimentally found, while the measured output coincidence distributions reach globally a similarity value of $97.5( \pm 1.3) \%$. 
(a)

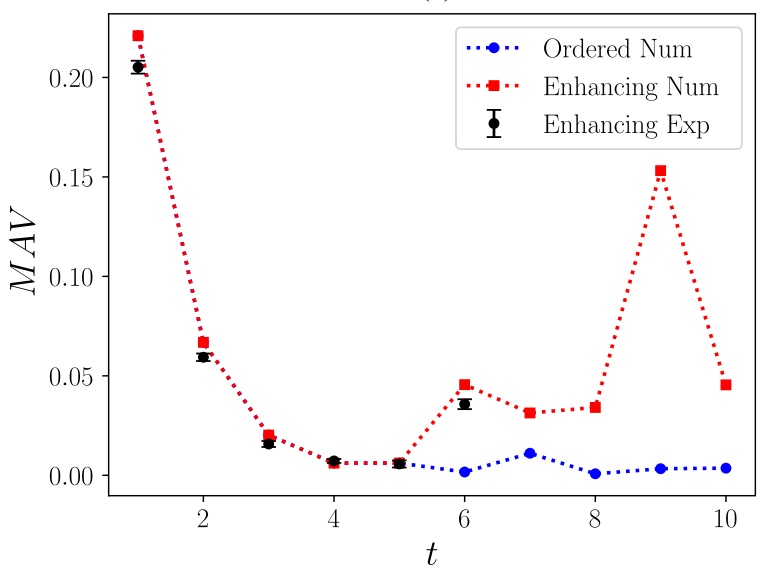

(b)

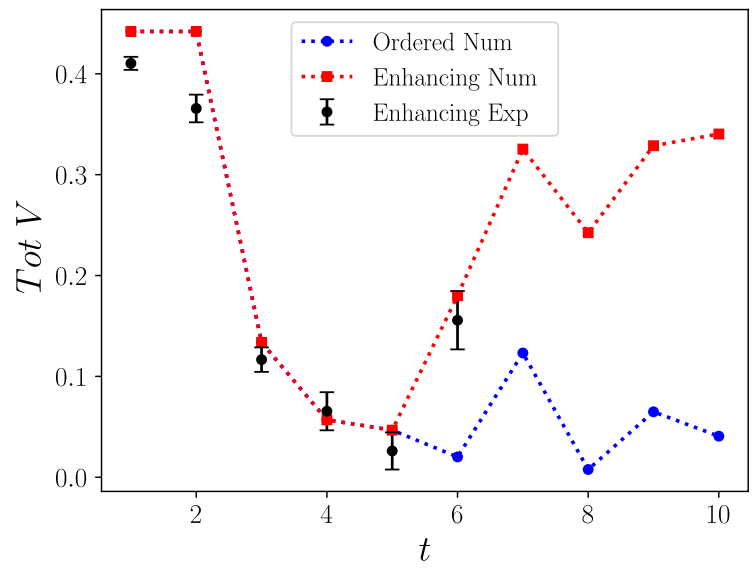

FIG. 8. Experimental results compression between the order and the enhancing disordered configuration $Q W$. Experimental results for (dark dot) (a) MAV and (b) corresponding total violation versus the number of steps. The trends are compared with the simulation for enhancing disorder (red squares) and ordered evolution (blue circles). The expected results are obtained by numerical simulations performed, accounting for experimental parameters and asymmetries, so that the theoretical trends show some discrepancies with respect to those in Fig. 5.

correlation spreads in a homogeneous network so that the values of $V_{i j}$ and the total violation are going to decline as the propagation proceeds. However, as can be seen in Fig. 8(a), the inhomogeneity enriches the quantum correlation between two indistinguishable photons at the given modes. Experimental evidences, reported in Fig. 8(a), show that the very same configuration also enhances the total quantum correlation of the QW. Experimental errors are derived from the Poissonian statistics of the measured coincidences. Deviations from the expected results are mainly due to modest drops in photons indistinguishability along the evolution. Nevertheless, the indistinguishability decline slightly affects the exact violation values, while not changing the overall trend.

\section{CONCLUSION}

The presented numerical and experimental analysis demonstrates that two-mode quantum correlations due to particle indistinguishability, which disperse through the lattice and rapidly decay in an ordered evolution, can be retrieved after a minimum evolution time by inserting suitable inhomogeneity patterns in the system. By changing the disorder configurations, it is possible to tune the two-mode and total enhancement of nonclassicality in position and intensity; this corresponds to an adaptive network whose parameters evaluation determines the focusing of nonclassical resources in selected modes. These findings allow for enriching quantum correlation through controlling unitary evolution (effective Hamiltonian engineering) of the biphoton. Also, we show that the two-mode quantum correlation diminishes in the case of random-phase disorder in the system, which can be interpreted as a manifestation of detrimental classical noise. Nevertheless, this quantum correlation degradation can be challenged by single realizations of disorder. These results supply a conceptual and practical advance compared to previous studies limited to single-photon disorder-assisted quantum correlation enhancement between two degrees of freedom of the photon [35]. In fact, since violations of Eq. (2) indicate biphoton quantum correlations between two modes, our method may also be interesting for quantum metrology issues. It is yet to be understood whether this enhancement procedure can be generalized to systems with $N>2$ photons or not, which could result in a benchmarking outcome in the context of quantum resource theories.

\section{ACKNOWLEDGMENTS}

We acknowledge support from the Italian project PRIN 2017 of MUR (Ministero dell'Università e della Ricerca) "Taming complexity via QUantum Strategies a Hybrid Integrated Photonic approach" (QUSHIP), Id. 2017SRNBRK. We also thank Fabio Sciarrino for useful discussions.

\section{APPENDIX A: QUANTITATIVE ANALYSIS OF VIOLATION}

The aim of this section is to provide a clearer interpretation of the quantity $V_{i j}$, which, in the main text, is exploited as a quantifier for nonclassicality in bosonic correlations. In particular, we focus on the relationship lying between this quantity and the two-particle boson bunching, the most simple and straightforward effect of bosonic indistinguishability; then, this relationship is extended to the network case, fitting the experimental implementation presented in the main text. As already described in the main text, the violation of the inequality:

$$
\frac{2}{3} \sqrt{\Gamma_{i, i} \Gamma_{j, j}}-\Gamma_{i, j}<0
$$

allows to point out the presence of nonclassical correlations in photonic systems $[48,50,52,53]$. The meaning of this simple relation can be traced back to the result of an elemental boson bunching phenomenon, i.e., the HOM effect [59], for the case of nonperfectly indistinguishable photons. We consider a photon pair with a given probability $q$ of being distinguishable, traveling through a supposedly balanced $B S$. After the $B S$, photons can be measured in three possible combinations of the two output modes and the probability of each combination depends on the probability of the photons being indistinguishable (which is $1-q$ ). Therefore, inequality (A1) 


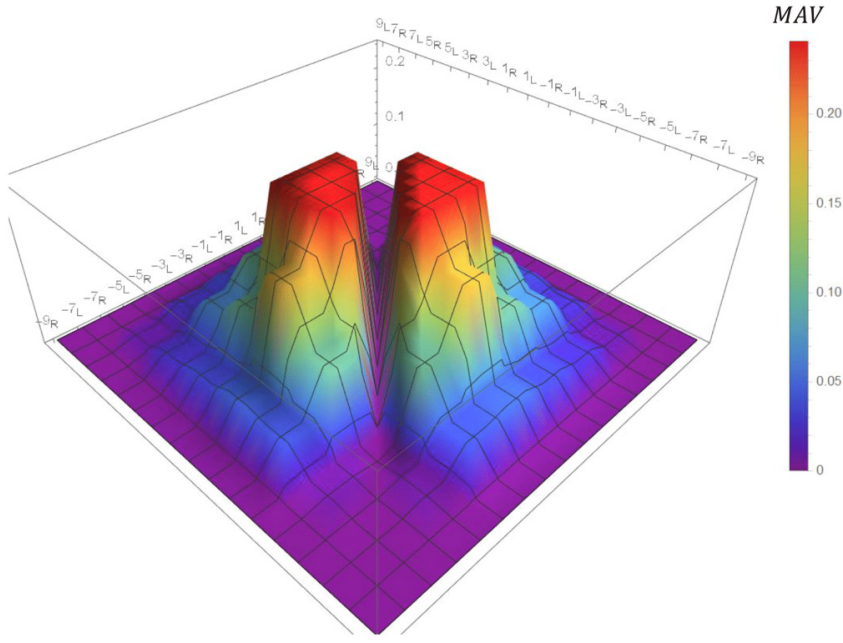

FIG. 9. Numerical simulation of 9 step MAV landscape. Plot of the maximum violation achievable for each output modes pair at step 9 obtained by comparing $10^{6}$ different disorder realizations.

can be written as:

$$
\frac{2}{3}\left(\frac{1}{2}-\frac{q}{4}\right)-\frac{q}{2}<0,
$$

which, in order to be violated, requires a value $q<\frac{1}{2}$, corresponding to photons that are more likely to be indistinguishable than distinguishable. Hence, the inequality (A1) provides a straightforward quantifier of the effective indistinguishability of photons, in the operational context of boson bunching occurrence. In the general case of a $B S$ network, such as the one exploited in the present work, a value of $V_{i j}>0$ can be subject to multiple interpretations. In the general case for a pure initial state, after an $n$ step propagation the system will be in a superposition state, which can be written considering the number of photons traveling in the modes of interest:

$$
\begin{aligned}
|\Phi\rangle= & \sqrt{1-\Pi}(\ldots)+\sqrt{\Pi}\left(\alpha_{k_{1} k_{1}}|2\rangle_{k_{1}}|0\rangle_{k_{2}}+\right. \\
& \left.+\alpha_{k_{1} k_{2}}|1\rangle_{k_{1}}|1\rangle_{k_{2}}+\alpha_{k_{2} k_{2}}|0\rangle_{k_{1}}|2\rangle_{k_{2}}\right),
\end{aligned}
$$

where $\Pi$ is the overall probability of having both photons in the selected modes, which normalizes the $\alpha_{i j}$ coefficients, while $\left\{\left|k_{1}\right\rangle,\left|k_{2}\right\rangle\right\}$ are the two output modes under observation, corresponding to combined states of position and coin of the form $|k\rangle:=|x\rangle|\sigma\rangle$. We do not consider contributions of other modes included in the first term (...). It is possible, obviously, to also have single-photon states of the two modes, but they would be invisible to coincidencelike measurements. In this case, the amount of violation between modes $k_{1}$ and $k_{2}$ can be computed as:

$$
V_{k_{1} k_{2}}=\Pi \times\left(\frac{2}{3} \sqrt{\left|\alpha_{k_{1} k_{1}}\right|^{2}\left|\alpha_{k_{2} k_{2}}\right|^{2}}-\left|\alpha_{k_{1} k_{2}}\right|^{2}\right) .
$$

Therefore, the violation amount depends on two factors:

- The actual nonclassicality of the correlation determining a positive or negative value.

- The global probability of the selected output modes (given by $\Pi$ ).
The first factor is the one pointing out the form of a hypothetically postselected state of the two photons emerging from the considered modes. The higher this factor, the cleaner the distillation of NOON states is by postselection, since it necessarily corresponds to a low $\left|\alpha_{k_{1} k_{2}}\right|^{2}$. The second factor is an amplification parameter, which gives the probability of actually finding two photons in the two-modes selected subsystem; hence, it gives the efficiency of the NOON states distillation. In conclusion, the violation value provides an indication over the composite effect of the two parameters; hence, its maximization can be related to either one or the other. Hence, this aspect needs to be taken into account in a hypothetical application of this protocols. For instance, the most external output modes will provide the most pure NOON states, since they are the mere propagation of the first HOM resulting state, but with a very low probability. On the other hand, by means of disorder, it is possible to manipulate the probability for central modes and get an higher efficiency, but at the cost of a nonzero chance of extracting a useless state.

\section{APPENDIX B: ADDITIONAL NUMERICAL RESULTS}

The highest MAV, besides the first step, is achieved at the output of the ninth step: The MAV for each modes pair $(i, j)$ of the ninth step output distribution was computed by analyzing $10^{6}$ different phase maps each. The resulting landscape in Fig. 9 shows that this maximum can be achieved in different positions, depending on the chosen enhancing disorder

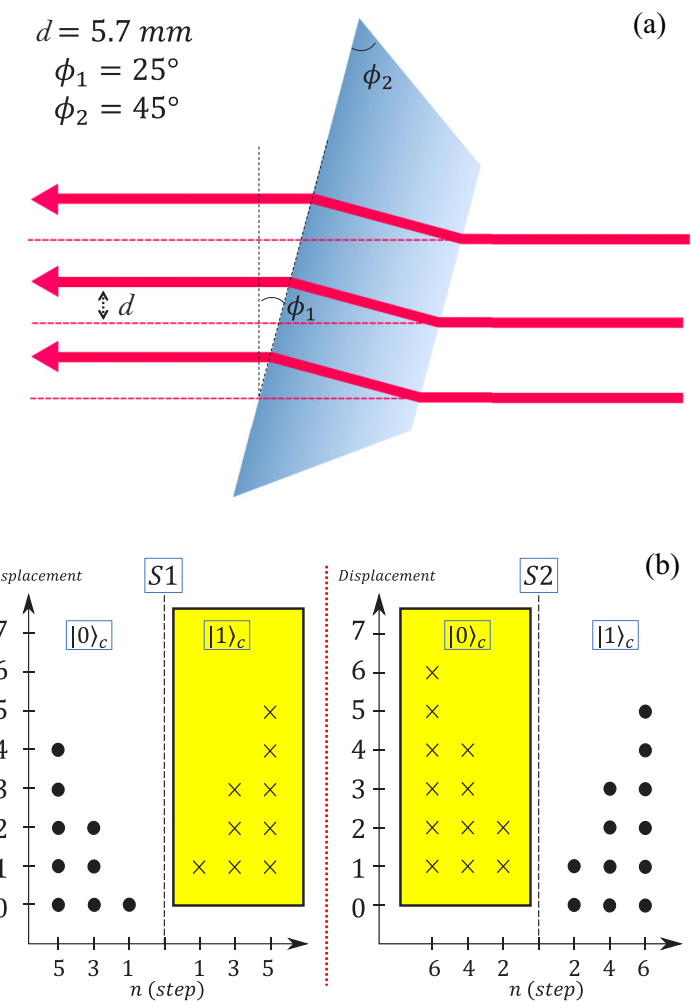

FIG. 10. BD and spatial structure of the $Q W$. (a) A sketch of the BD functioning, provided with relevant geometrical parameters. Through this device, it is possible to realize (b) a spatial structure featured by a distinct localization of each QW mode at any evolution step in the plane orthogonal to the propagation direction. 

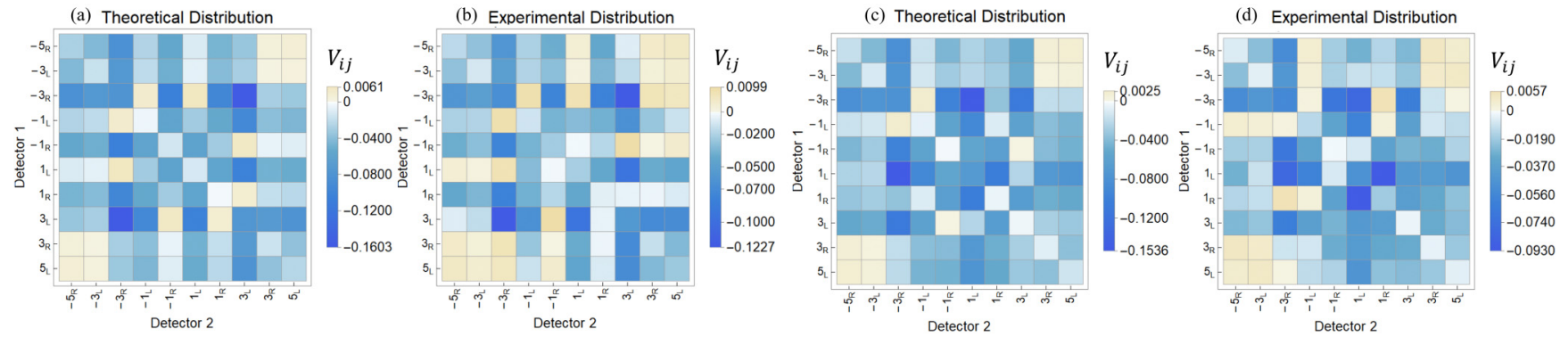

FIG. 11. Violation matrices at the fifth step. Theoretical and experimental violation matrices at the fifth step for [(a) and (b)] ordered evolution and [(c) and (d)] disordered evolution. The disorder configuration has been chosen randomly.

configuration. In particular, it suggests that the proper MAV can be achieved only in "central" modes pairs: The MAV can be induced between different mode pairs by imposing different (yet equally enhancing) phase maps, although that is not possible in modes that have not interfered enough. In fact, photons emerging from central modes will have the most interfering paths, becoming more affected by inhomogeneities along the evolution. This phenomenon is quite understandable considering the underlying network structure of the evolution (Fig. 1). Indeed, central modes are subjected to more complex interference phenomena with respect to those close to the boundaries, even more complex when their correlations are considered. This can be directly linked to the amount of MZIs jointly traveled by photons emerging from the two selected modes, i.e., the amount of phase shifts that are imposed over both photons. Therefore, regarding central modes, the manipulation of nonclassicality results is more powerful and effective.

\section{APPENDIX C: EXPERIMENTAL SETUP DETAILS}

The exploitation of the $z$ axis for the realization of the QW network relies on BDs Fig. 10(a), implementing the unique spatial distribution of the network nodes Fig. 10(b), essential to the actual realization of a space-time disorder. Photon pairs are generated by a high brilliance source realized according to the model described in Ref. [60]: A PPKTP crystal, embedded in a Sagnac interferometer, pumped by a CW laser radiation $\left(\lambda_{p}=405 \mathrm{~nm}\right)$, which generates a collinear pair of photons (idler and signal) with opposite polarization at a wavelength of $\lambda_{i, s}=2 \lambda_{p}=810 \mathrm{~nm}$. They are coupled to a pair of optical fibers and experience an additional path through air before starting the actual QW evolution. They are made indistinguishable in all possible degrees of freedom such as polarization, wavelength, and propagation mode. In particular, we tuned the relative free-space path to get the best bunching effect when they impinge on the bulk-optics BS (BS1 in Fig. 6) for the first time, corresponding to the first step of the QW. The unavoidable, critical free-space adjustment of beam superposition on the BS mainly limits the achieved visibility of HOM effect to an average $V \sim 89 \%$.

It is also worth mentioning the experimental method of measurement of the autocorrelation term $\Gamma_{i i}$ for each $i$ : A BS (BS2 in Fig. 6) placed at the output of the setup allows to split two photons traveling the same mode with probability $2 R T$ with $R(T)$ being the reflectivity (transmissivity) of the BS. By carefully characterizing the BS2 we can weigh the measured coincidences in order to compare them with the values of $\Gamma_{i j}$ for $j \neq i$. Our QW implementation is limited to a six-step evolution because of the internal losses in each unit passage (nearly 17\%) and because of both the geometrical structure and the length of the QW that make it difficult to guarantee a high quality spatial overlap of the two photons at each passage of light through the BS1. On the other hand, the main advantages of the QW configuration consist of both phase stability and a flexible disorder configurability and also by the fact that the output distribution for any step of the QW is directly accessible and measurable. As a consequence, this
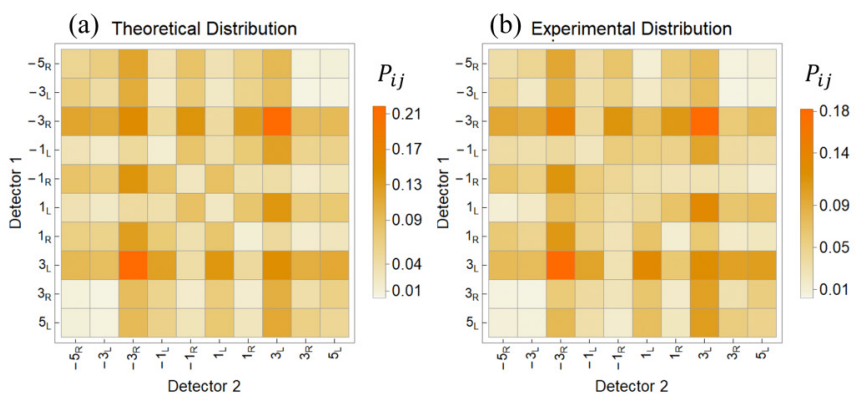

(c) Theoretical Distribution

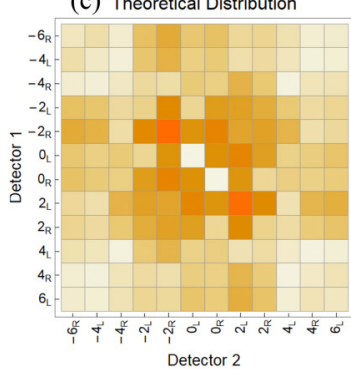

(d) Experimental Distribution

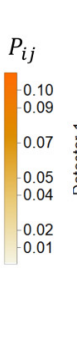

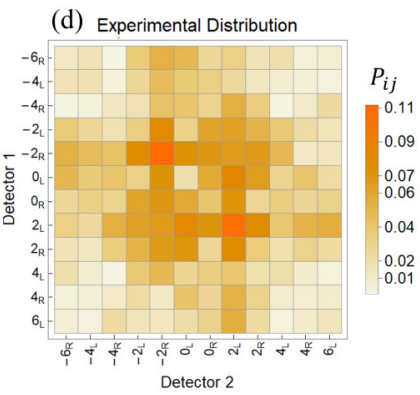

FIG. 12. Numerical and experimental coincidences matrices. Fifth step (a) theoretical coincidences matrix and (b) corresponding experimental measurement output for the ordered QW. Sixth step (c) theoretical coincidences matrix and (d) corresponding experimental measurement output for the optimal disordered configuration. The similarities between theoretical and experimental coincidences distributions are $98( \pm 1) \%$ for the fifth step case $(\mathrm{a}, \mathrm{b})$ and $97.5( \pm 1.3) \%$ for the sixth step case $(\mathrm{c}, \mathrm{d})$. Errors are computed accounting for Poissonian statistics of measured coincidences. 
QW platform is a powerful instrument for investigating the dynamical behavior of quantum features under any type of disorder.

An evolution of six steps through this setup is enough to investigate on the photon correlation enhancement effect.

Indeed, the numerical analysis reported in the main text indicates that a larger number of steps would not have brought any further physical insight into the experiment.

\section{APPENDIX D: ADDITIONAL EXPERIMENTAL RESULTS}

The output violation distributions corresponding to the fifth step are shown in Fig. 11. They provide a preliminary demonstration of the dependence of quantum correlation distributions from the disorder pattern imposed on the evolution. It is useful to observe the corresponding coincidence matrices for the ordered fifth step and the optimal sixth step (Fig. 12). Disorder, as demonstrated in several previous works $[43,44,46,60]$, determines first an effect of spread hindering, also for multiparticle systems. This effect can be noticed even in the case of a single disorder configuration (Fig. 12). The manipulation of this localization effect can change the nonclassicality pattern in the output distribution in many different configurations, changing the probability of finding coincident photons between the output modes. Indeed, the corresponding experimental coincidence distributions result in good agreement with the expected ones.
[1] W. H. Zurek, Decoherence, einselection, and the quantum origins of the classical, Rev. Mod. Phys. 75, 715 (2003).

[2] M. A. Nielsen and I. Chuang, Quantum Computation and Quantum Information (Cambridge University Press, Cambridge, England, UK, 2000).

[3] R. Horodecki, P. Horodecki, M. Horodecki, and K. Horodecki, Quantum entanglement, Rev. Mod. Phys. 81, 865 (2009).

[4] A. Streltsov, G. Adesso, and M. B. Plenio, Colloquium: Quantum coherence as a resource, Rev. Mod. Phys. 89, 041003 (2017).

[5] K. Modi, A. Brodutch, H. Cable, T. Paterek, and V. Vedral, The classical-quantum boundary for correlations: Discord and related measures, Rev. Mod. Phys. 84, 1655 (2012).

[6] M. T. Quintino, T. Vértesi, and N. Brunner, Joint Measurability, Einstein-Podolsky-Rosen Steering, and Bell Nonlocality, Phys. Rev. Lett. 113, 160402 (2014).

[7] H. M. Wiseman, S. J. Jones, and A. C. Doherty, Steering, Entanglement, Nonlocality, and the Einstein-Podolsky-Rosen Paradox, Phys. Rev. Lett. 98, 140402 (2007).

[8] N. H. Y. Ng and M. P. Woods, Resource theory of quantum thermodynamics: Thermal operations and second laws, in Thermodynamics in the Quantum Regime (Springer, Nature Switzerland AG, Cham, Switzerland, 2018), pp. 625-650.

[9] H. M. Wiseman and J. A. Vaccaro, Entanglement of Indistinguishable Particles Shared Between Two Parties, Phys. Rev. Lett. 91, 097902 (2003).

[10] R. Lo Franco and G. Compagno, Indistinguishability of Elementary Systems as a Resource for Quantum Information Processing, Phys. Rev. Lett. 120, 240403 (2018).

[11] Z. Ou, C. Hong, and L. Mandel, Relation between input and output states for a beam splitter, Opt. Commun. 63, 118 (1987).

[12] F. Nosrati, A. Castellini, G. Compagno, and R. Lo Franco, Robust entanglement preparation against noise by controlling spatial indistinguishability, npj Quantum Inf. 6, 39 (2020).

[13] T. Giordani, F. Flamini, M. Pompili, N. Viggianiello, N. Spagnolo, A. Crespi, R. Osellame, N. Wiebe, M. Walschaers, A. Buchleitner et al., Experimental statistical signature of manybody quantum interference, Nat. Photonics 12, 173 (2018).

[14] F. Benatti, R. Floreanini, F. Franchini, and U. Marzolino, Entanglement in indistinguishable particle systems, Phys. Rep. 878, 1 (2020).
[15] S. Bose and D. Home, Generic Entanglement Generation, Quantum Statistics, and Complementarity, Phys. Rev. Lett. 88, 050401 (2002).

[16] M. R. Barros, S. Chin, T. Pramanik, H.-T. Lim, Y.-W. Cho, J. Huh, and Y.-S. Kim, Entangling bosons through particle indistinguishability and spatial overlap, Opt. Express 28, 38083 (2020).

[17] K. Sun, Y. Wang, Z.-H. Liu, X.-Y. Xu, J.-S. Xu, C.-F. Li, G.-C. Guo, A. Castellini, F. Nosrati, G. Compagno et al., Experimental quantum entanglement and teleportation by tuning remote spatial indistinguishability of independent photons, Opt. Lett. 45, 6410 (2020).

[18] J. P. Dowling, Quantum optical metrology-the lowdown on high-NO0N states, Contemp. Phys. 49, 125 (2008).

[19] V. Giovannetti, S. Lloyd, and L. Maccone, Advances in quantum metrology, Nat. Photonics 5, 222 (2011).

[20] L. Mandel, Coherence and indistinguishability, Opt. Lett. 16, 1882 (1991).

[21] J. Sperling, A. Perez-Leija, K. Busch, and I. A. Walmsley, Quantum coherences of indistinguishable particles, Phys. Rev. A 96, 032334 (2017).

[22] A. Castellini, R. Lo Franco, L. Lami, A. Winter, G. Adesso, and G. Compagno, Indistinguishability-enabled coherence for quantum metrology, Phys. Rev. A 100, 012308 (2019).

[23] A. Perez-Leija, D. Guzmán-Silva, R. d. J. León-Montiel, M Gräfe, M. Heinrich, H. Moya-Cessa, K. Busch, and A. Szameit, Endurance of quantum coherence due to particle indistinguishability in noisy quantum networks, npj Quantum Inf. 4, 45 (2018).

[24] F. Nosrati, A. Castellini, G. Compagno, and R. Lo Franco, Dynamics of spatially indistinguishable particles and quantum entanglement protection, Phys. Rev. A 102, 062429 (2020).

[25] H.-K. Lo, M. Curty, and B. Qi, Measurement-DeviceIndependent Quantum Key Distribution, Phys. Rev. Lett. 108, 130503 (2012).

[26] Y. Liu, T.-Y. Chen, L.-J. Wang, H. Liang, G.-L. Shentu, J. Wang, K. Cui, H.-L. Yin, N.-L. Liu, L. Li et al., Experimental Measurement-Device-Independent Quantum Key Distribution, Phys. Rev. Lett. 111, 130502 (2013).

[27] S. Aaronson and A. Arkhipov, The computational complexity of linear optics, in Proceedings of the Forty-Third Annual ACM 
Symposium on Theory of Computing (Association for Computing Machinery, New York, NY, 2011), pp. 333-342.

[28] H.-S. Zhong, H. Wang, Y.-H. Deng, M.-C. Chen, L.-C. Peng, Y.-H. Luo, J. Qin, D. Wu, X. Ding, Y. Hu et al., Quantum computational advantage using photons, Science 370, 1460 (2020).

[29] V. Kendon, Decoherence in quantum walks—a review, Math. Struct. Comput. Sci. 17, 1169 (2007).

[30] M. Mohseni, P. Rebentrost, S. Lloyd, and A. Aspuru-Guzik, Environment-assisted quantum walks in photosynthetic energy transfer, J. Chem. Phys. 129, 11B603 (2008).

[31] V. Kendon and B. Tregenna, Decoherence can be useful in quantum walks, Phys. Rev. A 67, 042315 (2003).

[32] R. Vieira, E. P. Amorim, and G. Rigolin, Dynamically Disordered Quantum Walk as a Maximal Entanglement Generator, Phys. Rev. Lett. 111, 180503 (2013).

[33] R. Vieira, E. P. Amorim, and G. Rigolin, Entangling power of disordered quantum walks, Phys. Rev. A 89, 042307 (2014).

[34] M. Zeng and E. H. Yong, Discrete-time quantum walk with phase disorder: Localization and entanglement entropy, Sci. Rep. 7, 12024 (2017).

[35] Q.-Q. Wang, X.-Y. Xu, W.-W. Pan, K. Sun, J.-S. Xu, G. Chen, Y.-J. Han, C.-F. Li, and G.-C. Guo, Dynamic-disorderinduced enhancement of entanglement in photonic quantum walks, Optica 5, 1136 (2018).

[36] R. Lo Franco, B. Bellomo, E. Andersson, and G. Compagno, Revival of quantum correlations without system-environment back-action, Phys. Rev. A 85, 032318 (2012).

[37] J.-S. Xu, K. Sun, C.-F. Li, X.-Y. Xu, G.-C. Guo, E. Andersson, R. Lo Franco, and G. Compagno, Experimental recovery of quantum correlations in absence of system-environment backaction, Nat. Commun. 4, 2851 (2013).

[38] Y. Aharonov, L. Davidovich, and N. Zagury, Quantum random walks, Phys. Rev. A 48, 1687 (1993).

[39] A. Ambainis, E. Bach, A. Nayak, A. Vishwanath, and J. Watrous, One-dimensional quantum walks, in Proceedings of the thirty-third annual ACM symposium on Theory of computing (Association for Computing Machinery, New York, NY, 2001), pp. 37-49.

[40] S. E. Venegas-Andraca, Quantum walks: A comprehensive review, Quant. Info. Proc. 11, 1015 (2012).

[41] M. B. Plenio and S. F. Huelga, Dephasing-assisted transport: Quantum networks and biomolecules, New J. Phys. 10, 113019 (2008).

[42] V. B. Bulchandani, C. Karrasch, and J. E. Moore, Superdiffusive transport of energy in one-dimensional metals, Proc. Natl. Acad. Sci. U.S.A. 117, 12713 (2020).

[43] A. Schreiber, K. N. Cassemiro, V. Potoček, A. Gábris, I. Jex, and C. Silberhorn, Decoherence and Disorder in Quantum Walks: From Ballistic Spread to Localization, Phys. Rev. Lett. 106, 180403 (2011).

[44] A. Crespi, R. Osellame, R. Ramponi, V. Giovannetti, R. Fazio, L. Sansoni, F. De Nicola, F. Sciarrino, and P. Mataloni, Anderson localization of entangled photons in an integrated quantum walk, Nat. Photonics 7, 322 (2013).
[45] F. De Nicola, L. Sansoni, A. Crespi, R. Ramponi, R. Osellame, V. Giovannetti, R. Fazio, P. Mataloni, and F. Sciarrino, Quantum simulation of bosonic-fermionic noninteracting particles in disordered systems via a quantum walk, Phys. Rev. A 89, 032322 (2014).

[46] A. Geraldi, A. Laneve, L. D. Bonavena, L. Sansoni, J. Ferraz, A. Fratalocchi, F. Sciarrino, Á. Cuevas, and P. Mataloni, Experimental Investigation of Superdiffusion via Coherent Disordered Quantum Walks, Phys. Rev. Lett. 123, 140501 (2019).

[47] A. Geraldi, S. De, A. Laneve, S. Barkhofen, J. Sperling, P. Mataloni, and C. Silberhorn, Transient subdiffusion via disordered quantum walks, Phys. Rev. Research 3, 023052 (2021).

[48] Y. Bromberg, Y. Lahini, R. Morandotti, and Y. Silberberg, Quantum and Classical Correlations in Waveguide Lattices, Phys. Rev. Lett. 102, 253904 (2009).

[49] A. Peruzzo, M. Lobino, J. C. Matthews, N. Matsuda, A. Politi, K. Poulios, X.-Q. Zhou, Y. Lahini, N. Ismail, K. Wörhoff et al., Quantum walks of correlated photons, Science 329, 1500 (2010).

[50] Y. Lahini, M. Verbin, S. D. Huber, Y. Bromberg, R. Pugatch, and Y. Silberberg, Quantum walk of two interacting bosons, Phys. Rev. A 86, 011603(R) (2012).

[51] L. Sansoni, F. Sciarrino, G. Vallone, P. Mataloni, A. Crespi, R. Ramponi, and R. Osellame, Two-Particle Bosonic-Fermionic Quantum Walk via Integrated Photonics, Phys. Rev. Lett. 108, 010502 (2012).

[52] K. Poulios, R. Keil, D. Fry, J. D. A. Meinecke, J. C. F. Matthews, A. Politi, M. Lobino, M. Gräfe, M. Heinrich, S. Nolte, A. Szameit, and J. L. O'Brien, Quantum Walks of Correlated Photon Pairs in Two-Dimensional Waveguide Arrays, Phys. Rev. Lett. 112, 143604 (2014).

[53] Y. Lahini, Y. Bromberg, D. N. Christodoulides, and Y. Silberberg, Quantum Correlations in Two-Particle Anderson Localization, Phys. Rev. Lett. 105, 163905 (2010).

[54] R. Lo Franco and G. Compagno, Quantum entanglement of identical particles by standard information-theoretic notions, Sci. Rep. 6, 20603 (2016).

[55] G. Compagno, A. Castellini, and R. Lo Franco, Dealing with indistinguishable particles and their entanglement, Phil. Trans. R. Soc. A 376, 20170317 (2018).

[56] P. L. Knight, E. Roldán, and J. Sipe, Propagating quantum walks: The origin of interference structures, J. Mod. Opt. 51, 1761 (2004).

[57] H. Jeong, M. Paternostro, and M. S. Kim, Simulation of quantum random walks using the interference of a classical field, Phys. Rev. A 69, 012310 (2004).

[58] A. Geraldi, L. D. Bonavena, C. Liorni, P. Mataloni, and Á Cuevas, A novel bulk-optics scheme for quantum walk with high phase stability, Condens. Matter 4, 14 (2019).

[59] C.-K. Hong, Z.-Y. Ou, and L. Mandel, Measurement of Subpicosecond Time Intervals Between Two Photons by Interference, Phys. Rev. Lett. 59, 2044 (1987).

[60] A. Fedrizzi, T. Herbst, A. Poppe, T. Jennewein, and A. Zeilinger, A wavelength-tunable fiber-coupled source of narrowband entangled photons, Opt. Express 15, 15377 (2007). 